\title{
Development of Novel Animal Model for Studying Scoliosis Using a Noninvasive Method and Its Validation through Gene-Expression Analysis
}

\author{
Rajkiran Reddy Banala ${ }^{1}$, Satish Kumar Vemuri ${ }^{1}$, Murahari Penkulinti ${ }^{2}$, Gurava Reddy AV ${ }^{1}$, Subbaiah GPV ${ }^{1,2}$ \\ ${ }^{1}$ Sunshine Medical Academy of Research and Training, Sunshine Hospitals, Hyderabad, India \\ ${ }^{2}$ Department of Spine, Star Hospitals, Hyderabad, India
}

Study Design: To induce scoliosis in young female Wistar rats using a noninvasive method and to validate this model.

Purpose: To induce scoliosis in a rat model noninvasively by bracing and to study the corresponding gene-expression profile in the spine and different organs.

Overview of Literature: Scoliosis involves abnormal lateral curvature of the spine, the causes of which remain unclear. In the literature, it is suggested that scoliosis is genetically heterogeneous, as there are multiple factors involved directly or indirectly in its pathogenesis. Clinical and experimental studies were conducted to understand the etiology of anatomical alterations in the spine and internal organs, as the findings could help clinicians to establish new treatment approaches.

Methods: Twelve female Wistar rats aged 21 days were chosen for this study. Customized braces and real-time polymerase chain reaction (RT-PCR) primers for rats were designed using Primer 3 software. Radiological analysis (X-rays), histopathological studies, SYBR green, and RT-PCR analysis were performed.

Results: The spines of six rats were braced in a deformed position, which resulted in a permanent structural deformity as confirmed by X-ray studies. The remaining rats were used as controls. Quantitative studies of the expression of various genes (osteocalcin, pleiotrophins, matrix metalloproteinase-2 [MMP2] and MMP9, TIMP, interleukins 1 and 6, tumor necrosis factor- $\alpha$ ) showed their differential expression and significant upregulation $(p<0.05)$ in different organs of scoliotic rats in comparison to those in control rats. Histopathological findings showed tissue necrosis and fibrosis in the brain, retina, pancreas, kidney, liver, and disc of scoliotic rats. Conclusions: Bracing is a noninvasive method for inducing scoliosis in an animal model with $100 \%$ reliability and with corresponding changes in gene expression. Scoliosis does not just involve a spine deformity, but can be referred to as a systemic disease on the basis of the pathological changes observed in various internal organs.

Keywords: Adolescent idiopathic scoliosis; Noninvasive method; Inflammation; Histopathology

\section{Introduction}

Scoliosis involves abnormal lateral curvature of the spine, with the term derived from the Greek word skoliosis meaning bending [1]. The diagnosis of scoliosis is performed through anteroposterior plain radiography, with the definition referring to curves $>10^{\circ}$ [2]. The causes of scoliosis are still unclear, but some neuromuscular condi-

Received May 8, 2018 ; Revised Jun 18, 2018; Accepted Jun 27, 2018

Corresponding author: Subbaiah GPV

Sunshine Medical Academy of Research and Training, Sunshine Hospitals, Hyderabad, Telangana, India; Department of Spine, Star Hospitals, Road No 10, Banjara Hills, Hyderabad, Telangana, India

Tel: +91-040-44 777 777, Fax: +91-040-2335 6188, E-mail: drgpvsubbaiahgoli@gmail.com 
tions, such as cerebral palsy and muscular dystrophy, and developmental malformations of the vertebral bodies, such as hemivertebrae and unsegmented bars, could be involved in some patients [3].

In the literature, it is suggested that scoliosis is genetically heterogeneous, as there are multiple factors involved dependently or independently in its pathogenesis. Adolescent idiopathic scoliosis (AIS) is believed to be multifactorial; so the identification of genes playing a role in its incidence could help clinicians and researchers to understand this common spinal deformity, which may lead to new therapeutic options [1-3].

Clinical and experimental studies have been performed to elucidate the anatomical alterations in the spine and other internal organs associated with scoliosis, which might help to shed light on the etiology and establish new treatment approaches [3-7]. In this context, several experimental animal models have been developed over the years by various research groups all over the world, examples of which are as follows.

(1) Ablation of pineal gland or hypophysis/pituitary gland in developing chickens (i.e., white Leghorn chickens).

(2) Development of melatonin-knockout mice (congenital melatonin deficiency) and reversal of scoliosis development by the daily administration of melatonin.

(3) Development of pinealectomized bipedal mice and rats (the induction rate of scoliosis=70\%)

(4) Administration of beta-aminopropionitrile and semicarbazide in pinealectomized bipedal rats also resulted in scoliosis and alteration in the vertebral ligaments [8-11].

(5) Development of pinealectomized fish to study spinal deformity or curvature

(6) In bipedal non-human primate models, the development of scoliosis was not observed despite a significant drop in melatonin levels [12].

All of the above mentioned scoliotic animal models were developed by using invasive methods; moreover, replicating these models could be difficult as their success rate is limited to $70 \%$ and they are associated with some mortality. However, apart from a couple of exceptions, no major studies inducing scoliosis in animals through noninvasive methods have been performed. These exceptions include the work of Grivas et al. [13] in 2011, who used a mini-Ilizarov external fixator, which is a semi-invasive method, for inducing scoliosis in Wistar rats. Moreover, Silva et al. [14] in 2012 used a simple bracing technique for inducing scoliotic curves in rats. However, none of these groups performed any gene expression or histopathological studies to understand the etiology of scoliosis.

In the present work, we studied the gene-expression profile of different genes implicated in the etiology of scoliosis and histological alterations in various internal organs associated with this condition. The aims here were to induce scoliosis through noninvasive methods, to study a few genes (pleiotrophins [PTNs], TIMPs, matrix metalloproteinase-2 [MMP2] and MMP9, interleukin-1 [IL1], IL6, tumor necrosis factor- $\alpha$ [TNF- $\alpha$ ], and osteocalcin) putatively believed to play a pivotal role in the etiology of scoliosis, and to determine whether any alterations in their gene-expression patterns occur with external bracing alone.

PTNs belong to the family of heparin-binding growth factors. They can enhance angiogenesis, bone repair, and bone remodeling through their receptor protein tyrosine phosphatase beta/zeta (RPTPb/f). In the literature, it is suggested that enhanced PTN expression occurs in serum, cartilage, and subchondral bone of osteoarthritis patients $[13,14]$. Another study showed that mechanical loading on the spine can initiate the expression of PTN [15]. Osteocalcin and RANK genes are involved in bone-remodeling changes. The induction of scoliosis by external pressure may initiate the inflammatory cytokinerelated response (by ILs and TNF- $\alpha$ ) and the activation of other matrix metalloproteases (MMPs and TIMPs) as well [7,16-21].

\section{Materials and Methods}

Female Wistar rats aged 21 days were procured from Jeeva Life Sciences Pvt. Ltd. (Hyderabad, India). The bracing material (i.e., leather) and LED light joint wires were purchased from local markets. Rat spines were braced in a scoliotic position. Real-time polymerase chain reaction (RT-PCR) primers for rat were designed using Primer 3 software for the targeted genes and procured from Eurofins Genomics India Pvt. Ltd. SYBR Premix Ex Taq, TaKaRa BioMasher Standard Verso cDNA synthesis kit (Thermo Scientific, Waltham, MA, USA; cat. no. AB1453/A), Seakem LE agarose (Lonza, Basel, Switzerland; cat. no. 50004), RNA Sure mini kit (Nucleopore, Genetix, 
India; cat. no. NP-84105), and Insta Q96 RT-PCR Machine (HiMedia Pvt. Ltd., Mumbai, India) were used for gene-expression studies.

\section{Noninvasive method}

1) Bracing for partial immobilization and radiological findings

Twelve female Wistar rats were divided equally into two groups with the same age distribution. Scoliosis was induced in six rats by the progressive application of a spinal brace in a deformed position, whereas the other six rats were used as controls (Animal Ethical Committee permission no. CPCSEA/IAEC/JLS/003/03/15/001). The leather braces were customized as per our requirements and were knotted using LED light joint wires. The experiment lasted 7 weeks, during which the rats were braced for 5 weeks and the braces were replaced every week in line with the rats' growth. As a result of bracing, the rats were partially immobilized and their spines were curved at angle of $40^{\circ}-50^{\circ}$. The bracing was maintained for 5 weeks and then the rats were left unbraced for 2 weeks to check whether the curve formed in the spine because bracing was permanent. Whole-spine X-rays (X-ray exposure: 800 MA, Polydros Lix, $46 \mathrm{KV}, 5 \mathrm{MAS}$ ) were taken every week until the end of the experiment to check the progression of curving or bending in the thoracic region of the spine. After 7 weeks, the rats were sacrificed by cervical dislocation. All relevant organs (brain, retina, liver, pancreas, kidney, and spine) were collected from rats in RNA-later solution and stored at $-80^{\circ} \mathrm{C}$ for further analysis. Part of the tissue of each organ was fixed in paraformaldehyde for histopathological studies (Fig. 1).

\section{2) Total RNA purification and cDNA synthesis}

The total RNA was isolated from various organs (brain, retina, liver, pancreas, kidney, and spine) using a commercially available kit (RNA Sure mini kit, Nucleopore; NP-84105) and the purity of RNA was analyzed using Nanodrop. cDNA synthesis was performed using a commercially available kit (Verso cDNA synthesis kit, Thermo Scientific) in two steps: at $42^{\circ} \mathrm{C}$ for 30 minutes (synthesis step) and at $95^{\circ} \mathrm{C}$ for 2 minutes (inactivation step). Five ng of total RNA was used as a template.

3) Semi-quantitative real-time polymerase chain reaction Total RNA was isolated from various tissues (brain, retina,

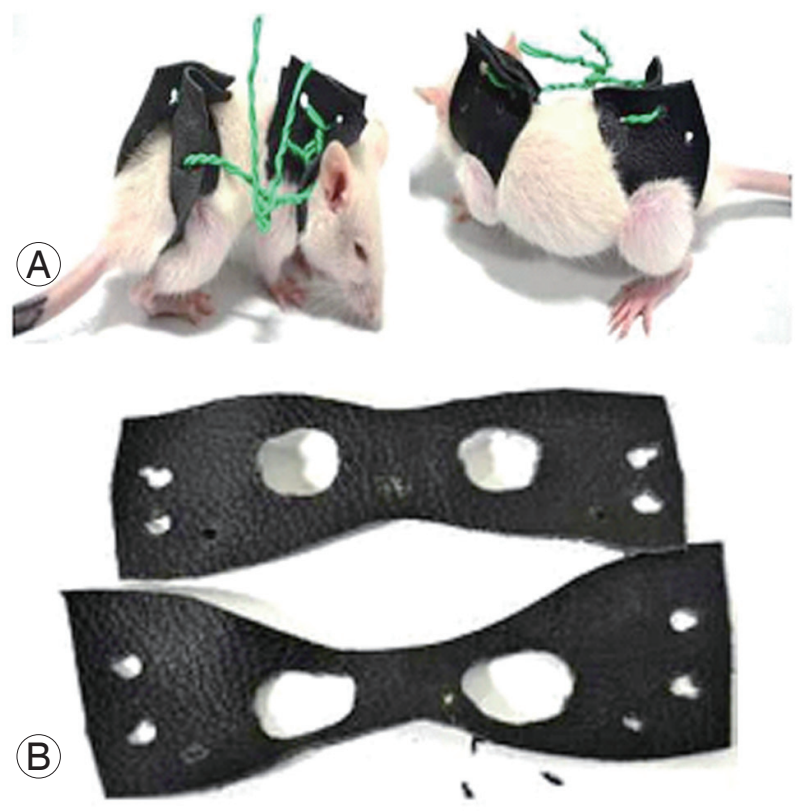

Fig. 1. (A, B) Braces and bracing of young rats.

liver, pancreas, kidney, and spine) using RNA Sure mini kit (Nucleopore), as described by the manufacturer. RTPCR was performed sequentially in the same $0.5-\mathrm{mL}$ RNase-free tubes under optimized conditions, and all RTPCR reagents were purchased from Takara (India). A total of $10 \mu \mathrm{L}$ of SYBR Premix Ex Taq (TliRNaseH Plus, $2 \times$ ), 0.4 $\mu \mathrm{L}$ of forward primer $(10 \mu \mathrm{M})$ and reverse primer $(10 \mu \mathrm{M}$, Eurofins Pvt. Ltd.), $0.4 \mu \mathrm{L}$ of ROX Reference Dye $(50 \times)$ or Dye II $(50 \times)$, and $0.2 \mu \mathrm{L}$ of template (cDNA) were included in a $20-\mu \mathrm{L}$ reaction mixture by adding $6.8 \mu \mathrm{L}$ of sterile $\mathrm{dH}_{2} \mathrm{O}$. Cycle parameters were generally a 10 -minute melting step at $95^{\circ} \mathrm{C}$, followed by 30 seconds at $95^{\circ} \mathrm{C}$, a 1 -minute annealing step at various temperatures $\left(55^{\circ} \mathrm{C}-61^{\circ} \mathrm{C}\right)$ for different genes, and a 1 -minute extension step at $72^{\circ} \mathrm{C}$. A total of 40 cycles were selected for osteocalcin, MMP2, MMP9, TIMP-2, and PTN (PTNs and mPTN) mRNA amplification, respectively (Table 1).

\section{Histopathology}

Tissue samples were collected in $10 \%$ formalin for histopathological studies and embedded using paraffin. Fivemicron-thick sections were produced and stained with hematoxylin and eosin. Light microscopic examination of multiple tissue sections from each organ was performed in all groups and images representative of the typical histological profile were examined. 
Table 1. List of hypothetical genes and primer sequences

\begin{tabular}{|c|c|c|c|}
\hline \multirow{2}{*}{ Gene name } & \multicolumn{2}{|c|}{ Primer sequence } & \multirow{2}{*}{ - Melting point $\left({ }^{\circ} \mathrm{C}\right)$} \\
\hline & Forward & Reverse & \\
\hline Osteocalcin & AAGCCCAGCGACTCTGAGTCT & GCTCCAAGTCCATTGTTGAGGTA & 61 \\
\hline mPTN & AAAAATGTCGTCCCAGCAAT & GCTCСАААСTGCTTCTTCCA & 55 \\
\hline PTN & GACTCAGAGATGTAAGATCCC & TGTGCAGAGCTCTCTTCAGAC & 57 \\
\hline TIMP2 & GTTTTGCAATGCAGATGTAG & ATGTCGAGAAACTCCTGCTT & 58 \\
\hline MMP2 & CTGATAACCTGGATGCAGTCGT & CCAGCCAGTCCGATTTGA & 58 \\
\hline MMP9 & TTCAAGGACGGTCGGTATT & CTCTGAGCCTAGACCCAACTTA & 56 \\
\hline IL1 & CAC CTT CTT TTC CTT CAT CTT TG & GTC GTT GCT TGT CTC TCC TTG TA & 58 \\
\hline IL6 & TGA TGG ATG CTT CCA AAC TG & GAG CAT TGG AAG TTG GGG TA & 56 \\
\hline TNF- $\bigotimes$ & ACT GAA CTT CGG GGT GAT TG & GCT TGG TGG TTT GCT ACG AC & 58 \\
\hline GAPDH & TGAGGTGACCGCATCTTCTTG & TGGTAACCAGGCGTCCGATA & 59 \\
\hline
\end{tabular}

PTN, pleiotrophin; MMP2, matrix metalloproteinase-2; MMP9, matrix metalloproteinase-9; IL, interleukin; TNF, tumor necrosis factor; GAPDH, glyceraldehyde-3-phosphate dehydrogenase.

\section{Statistical analysis}

The data are presented as mean \pm standard error of the mean (SEM). The data were statistically analyzed by twoway analysis of variance (ANOVA) to determine the effects of all groups on each of the experimental parameters, and Student $t$-test was used to compare the means of each parameter between the groups. A mean difference was considered significant at a $p$-value of $<0.05$. Statistical analysis was performed using IBM SPSS for Windows ver. 20.0 (IBM Corp., Armonk, NY, USA).

\section{Results}

\section{Radiographical findings}

Weekly spinal radiographs of the rats showed the development of scoliosis in the study group because of bracing (5 weeks). The persistence of spinal deformity was observed even after 2 weeks of unbracing. At the end of 5 weeks of bracing, an average curve of $40^{\circ}$ was noted, which was relatively well maintained at the end of 7 weeks at $30^{\circ} \pm 5^{\circ}$. This proves that one can induce scoliosis in animal models through noninvasive methods.

$\mathrm{X}$-rays of control and braced rats were taken weekly (i.e., for 7 weeks) to monitor the spinal deformity produced by bracing. Braces were changed every week to allow the rats to undergo normal growth and reach a normal size. Xrays of the experimental group were obtained for 5 weeks

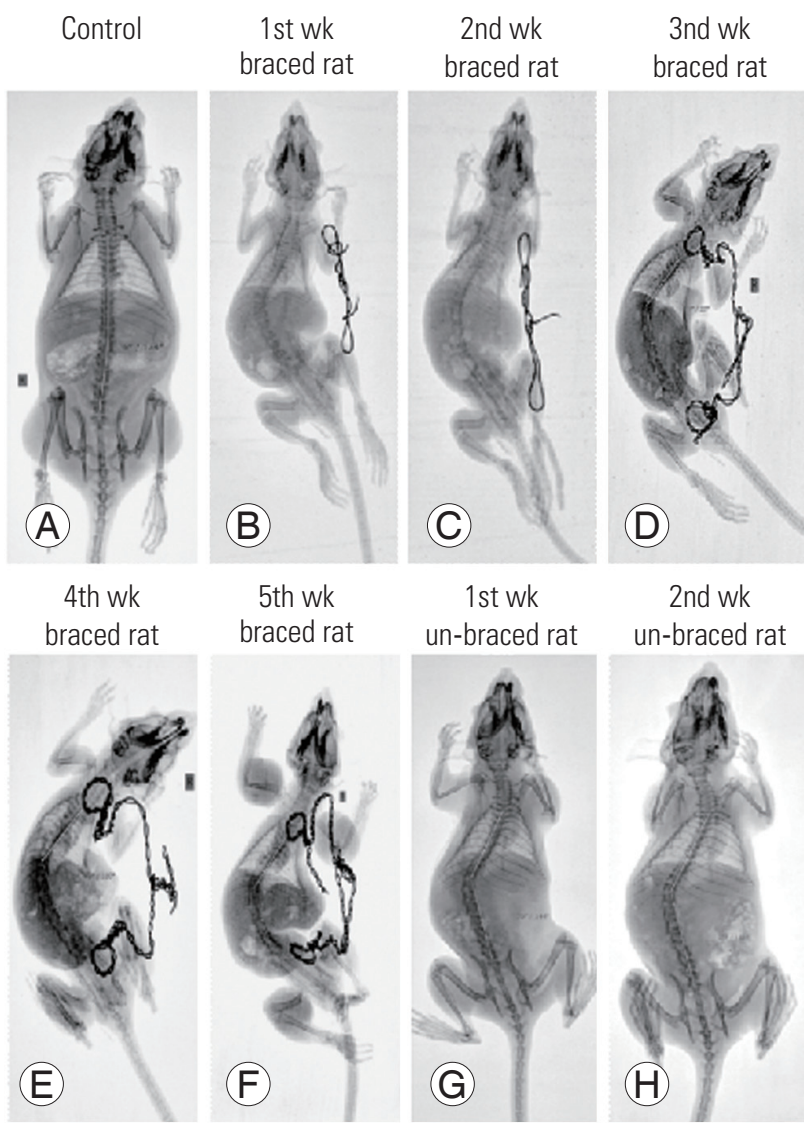

Fig. 2. (A-H) Radiographs of young rats and progression of scoliosis.

with braces and the next 2 weeks without them. The radiographical findings revealed that the spinal deformity persisted even after the bracing had been removed (Fig. 2). 
Table 2. Histological findings from different organs

\begin{tabular}{|c|c|c|c|}
\hline Panel no. of Fig. 3 & Organ & Control sample & Scoliotic sample \\
\hline A & Spine & No degeneration & $\begin{array}{l}\text { Moderate degeneration/demyelination surrounding } \\
\text { central vein }\end{array}$ \\
\hline B & Brain & $\begin{array}{l}\text { No degeneration } \\
\text { Moderate proliferation of ependymal cells in brain } \\
\text { ventricles }\end{array}$ & $\begin{array}{l}\text { Foci of necrosis along with neural death in cerebral } \\
\text { hemespheres }\end{array}$ \\
\hline C & Liver & Normal hepatocytes & Sinusoidal hemorrhages (black arrow) \\
\hline D & Kidney & Normal morphology & Tubular hemorrhages with inflammation \\
\hline E & Pancreas & Acinar cells normal & $\begin{array}{l}\text { Non glandular region shows periductular fibrosis } \\
\text { (black arrow) with inflammation (red arrow) }\end{array}$ \\
\hline $\mathrm{F}$ & Retina & Lens, retina, cornea show normal morphology. & Fibrosis surrounding cilliary eye muscle \\
\hline G & Heart & $\begin{array}{l}\text { Normal morphology of cardiomyocytes, myocardial and } \\
\text { pericardial layer }\end{array}$ & $\begin{array}{l}\text { Plaque deposition n coronary artery (black arrow) } \\
\text { Inflammation in external layer of aorta (red arrow) }\end{array}$ \\
\hline
\end{tabular}
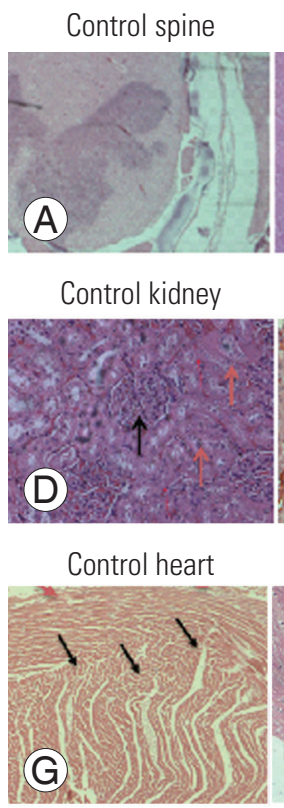

Induced spine

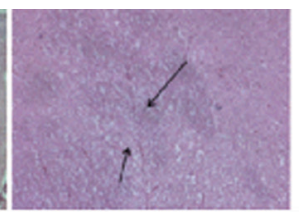

Induced kidney

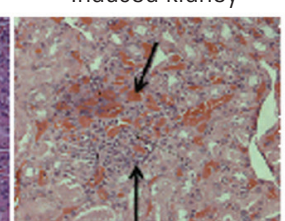

Induced heart

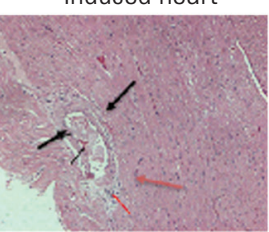

Control brain

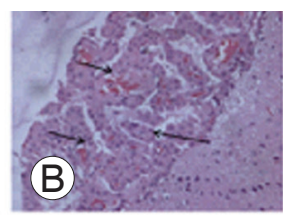

Control pancreas

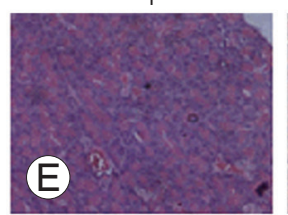

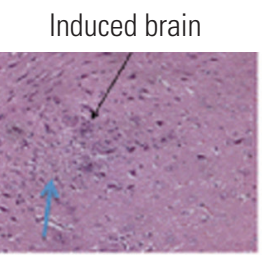

Induced pancreas

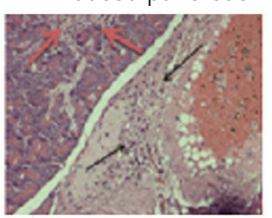

Control liver

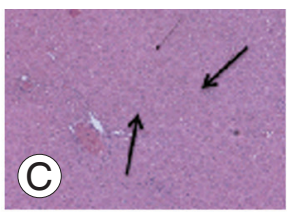

Control retina

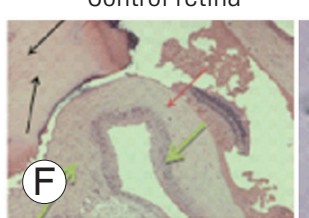

Induced liver

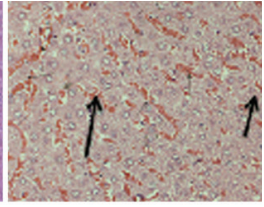

Induced retina

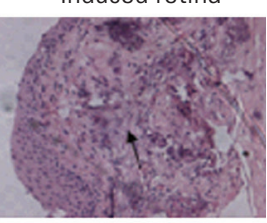

Fig. 3. (A-G) Histopathological findings on various organs (arrows).

The histopathological findings from the different organs collected from normal and scoliotic samples as reported here were obtained from the above images (Table 2, Fig. 3).

\section{Semi-quantitative real-time polymerase chain reac- tion analysis}

The housekeeper gene GAPDH (glyceraldehyde-3phosphate dehydrogenase) was used as a reference control and showed no difference in expression between the study group and control group. The expression of different genes, such as PTNs, mPTN, TIMP2, MMP2, MMP9, IL1, IL6, TNF- $\alpha$, and osteocalcin, was also analyzed in each organ.

Two-way ANOVA was performed by comparing the expression profile of different genes in both controls and induced samples (4A-F) (Table 3$)$. The values were plotted as mean $\pm \operatorname{SEM}(p<0.05$, statistically significant).

The fold change of expression of different genes in each organ of control and scoliotic rats was analyzed. The control samples are presented in blue and the scoliotic samples in orange. The above graphs were prepared by comparing the fold change values observed in controls and in samples with induced scoliosis. Fig. 4 and Table 3 clearly indicate the differential gene expression in each organ of the scoliotic rats in comparison with the gene expression 

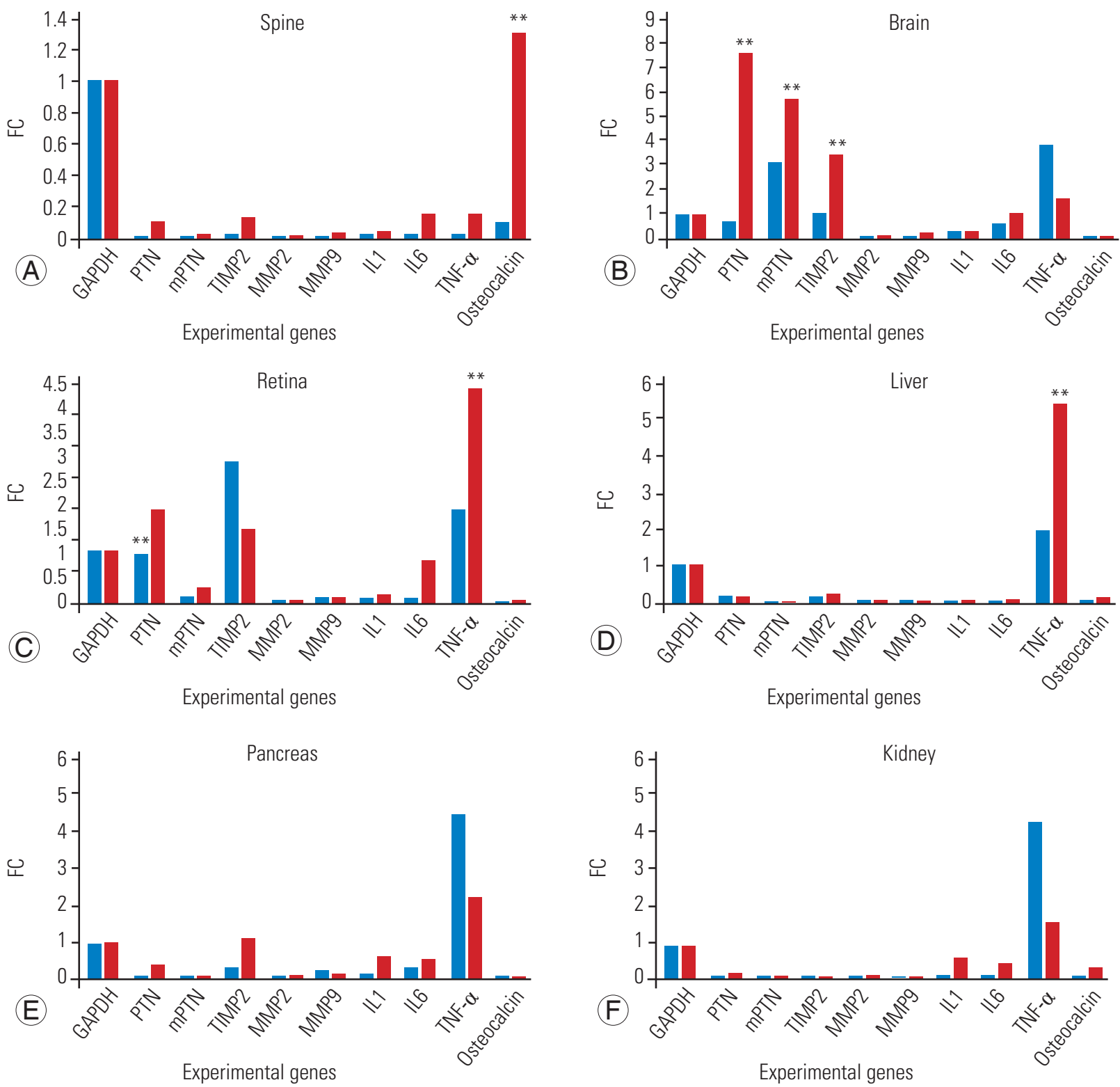

Control-FC

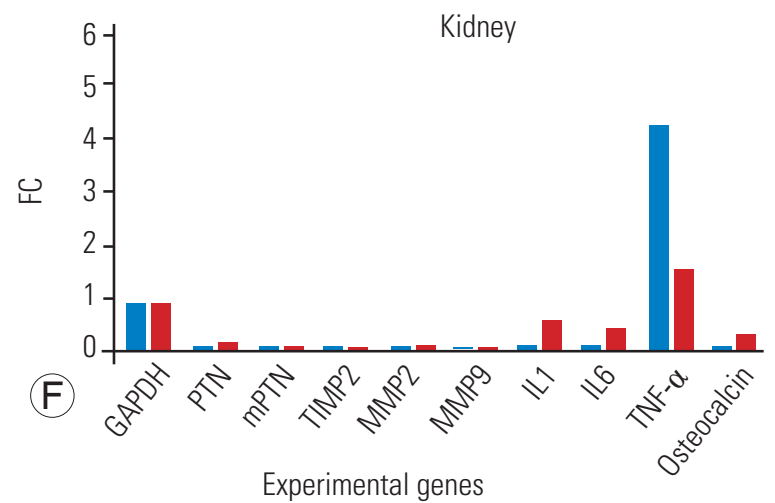

Induced-FC

Fig. 4. (A-F) Quantitate real-time polymerase chain reaction analysis of gene expression of different genes in varied organs. GAPDH, glyceraldehyde-3-phosphate dehydrogenase; PTN, pleiotrophin; MMP2, matrix metalloproteinase-2; MMP9, matrix metalloproteinase-9; IL, interleukin; TNF, tumor necrosis factor; FC, fold change.

observed in the control rats.

\section{Discussion}

Scoliosis is a complex four-dimensional deformity of the spine [1-3]. Although it is the most common spinal deformity, the majority of cases are idiopathic [1]. Our understanding of scoliosis has changed over time, and from a simple coronal plane deformity, it is now known to involve a four-dimensional deformity, with deformities in the coronal plane, sagittal plane, axial plane, and chest wall as the fourth plane [4-6]. Researchers have induced scoliosis in various animal models to understand the etiology of AIS; the well-known models are quadrupedal (mechanical and genetic models) and bipedal (mechanical and neuroendocrine models) [8-12,22]. The axial loading and dorsal shear stress exerted in bipeds differ from those in quadrupeds [12]. Nonetheless, quadrupeds are the 
Table 3. Expression profile of hypothetical genes in different organs of scoliotic rats in comparison to the control rats

\begin{tabular}{|c|c|c|c|c|c|c|}
\hline Gene & Spine & Retina & Brain & Liver & Kidney & Pancreas \\
\hline Osteocalcin & Upregulation & Down regulation & Down regulation & Down regulation & Upregulation & Down regulation \\
\hline mPTN & Upregulation & Upregulation & Upregulation & Down regulation & Down regulation & Down regulation \\
\hline PTN & Upregulation & Upregulation & Upregulation & Down regulation & Upregulation & Upregulation \\
\hline TIMP2 & Upregulation & Down regulation & Upregulation & Upregulation & Down regulation & Upregulation \\
\hline MMP2 & Down regulation & Down regulation & Down regulation & Down regulation & Down regulation & Down regulation \\
\hline MMP9 & Upregulation & Down regulation & Upregulation & Down regulation & Down regulation & Down regulation \\
\hline IL1 & Upregulation & Upregulation & Upregulation & Upregulation & Upregulation & Upregulation \\
\hline IL6 & Upregulation & Upregulation & Upregulation & Upregulation & Upregulation & Upregulation \\
\hline TNF- $\alpha$ & Upregulation & Upregulation & Down regulation & Upregulation & Down regulation & Down regulation \\
\hline
\end{tabular}

PTN, pleiotrophin; MMP2, matrix metalloproteinase-2; MMP9, matrix metalloproteinase-9; IL, interleukin; TNF, tumor necrosis factor.

models most widely used for studying scoliosis. The mechanical models have failed to provide significant inputs into the etiology of idiopathic scoliosis, but they can help in designing new treatment modalities.

The primary techniques employed in quadrupedal animals for inducing scoliosis include (1) the resection of ribs; (2) the excision or denervation of local musculature; or (3) the fixation of vertebrae or ribs to each other. The spine curvature induced in animal models is consistent with clinical evidence that prolonged recumbence can also cause scoliotic deformity, negating the gravity hypothesis suggesting that gravity is not in fact a prerequisite. To date, the majority of experiments on the induction of scoliosis in animals have followed these techniques, but none of the above-discussed methods achieved results that were $100 \%$ reproducible [8-12].

Studies aimed at understanding the etiopathogenesis of this deformity have suggested a multifactorial cause with a strong genetic predisposition. Nervous system, hormonal, biochemical, musculoskeletal, and environmental factors have been suggested to contribute to the genesis of scoliosis [6,7,16-21]. None of these factors is exclusive to the spine and it is possible that the same factors can exert deleterious effects on other organs; therefore, we can define scoliosis as a systemic disease. Isolated effects of scoliosis on other organs have been studied. Burner et al. [23] reported the presence of generalized osteopenia in AIS. Moreover, in a study involving 198 girls with AIS, Lee et al. [22] found a strong association between IL-6 gene polymorphism and osteopenia. Anatomical abnormalities in various parts of the brain and spine have also been reported [8-10]. Moreover, neurophysiological pathology has also been noted, including oculovestibular dysfunction and postural imbalance $[17,18]$. The levels of hormones such as melatonin, leptin, and calmodulin have also been found to be altered in patients with scoliosis and have been studied to identify associations between hormonal dysfunction and the genesis of scoliosis [1619]. Studies have also shown the existence of paraspinal muscle fibrosis in AIS [16].

There are several invasive methods that can induce scoliosis in animal models, but only one or two noninvasive equivalents exist, for which the rate of induction was very low. The noninvasive methods developed by Grivas et al. [13] in 2011 and Silva et al. [14] in 2012 were successful at inducing spinal deformity in rat models, but the rate of induction of scoliosis was approximately $50 \%$ and they did not study or confirm histological alterations in internal organs. Moreover, no studies of gene expression were performed in multiple organs in scoliotic animals.

We hypothesized that constant external pressure through bracing in a scoliotic position could induce structural scoliosis and pathological changes in internal organs (brain, retina, liver, pancreas, and kidney), which is proved by the cellular changes (histopathological and gene-expression studies) observed in the present study.

The histological findings showed focal necrosis in tissues such as brain, retina, liver, pancreas, and kidney. The gene-expression profile of PTNs, TIMP2, MMP2, MMP9, IL1, IL6, TNF- $\alpha$, and osteocalcin in all tissue samples was analyzed and found to be statistically significant in comparison to the expression pattern observed in the control group. There was significant upregulation in the expression of PTNs and interleukins in spine and all other or- 
gans, but the expression profile of matrix metalloproteases (MPP2, MPP9, and TIMP2), TNF- $\alpha$, and osteocalcin was found to vary from organ to organ in the experimental models.

The observations performed in this study are useful for understanding the effects of scoliosis on multiple organs, but further studies are required to elucidate the cascade of events that lead to such damage and how individual factors are associated with such damage or deformity. Scoliosis is known as a spinal disorder, but according to the findings in various internal organs, we should focus on studying its various effects on the internal organs.

\section{Conclusions}

Although animal models may not replicate the human spine, they remain the best available modality to study scoliosis. We successful devised an easy, noninvasive, and cost-effective method to induce scoliosis with $100 \%$ accuracy in rats, unlike previously described invasive methods of inducing it. This model was further validated through gene-expression profiling, which showed significant upregulation of implicated genes in the spine and other internal organs as a result of constant exposure to external pressure in a scoliotic position.

Our findings suggest future directions as follows. (1) Epigenetic changes and their role in scoliosis can be studied. (2) Alterations in neurotransmitters have been reported in scoliosis patients, so dietary modifications could modulate the levels of neurotransmitters in the body, which could be helpful in the recovery from curvature and correction of spinal deformity. (3) Structural/ functional changes in the brain and other internal organs associated with scoliosis can be studied by advanced imaging techniques.

\section{Conflict of Interest}

No potential conflict of interest relevant to this article was reported.

\section{Acknowledgments}

We wish to extend our sincere thanks to all individuals who supported us with the analysis and experimentation. We especially thank HiMedia Pvt. Ltd., India, for help with the RT-PCR analysis.

\section{References}

1. Nogami H, Terashima Y, Tamaki K, Oohira A. Congenital kyphoscoliosis and spinal cord lesion produced in the rat by beta-aminopropionitrile. Teratology 1977;16:351-7.

2. Beguiristain JL, De Salis J, Oriaifo A, Caaadell J. Experimental scoliosis by epiphysiodesis in pigs. Int Orthop 1980;3:317-21.

3. Tanaka H, Kimura Y, Ujino Y. The experimental study of scoliosis in bipedal rat in lathyrism. Arch Orthop Trauma Surg 1982;101:1-27.

4. Dabney KW, Salzman SK, Wakabayashi T, et al. Experimental scoliosis in the rat: II. biomechanical analysis of the forces during Harrington distraction. Spine (Phila Pa 1976) 1988;13:472-7.

5. Kasuga K. Experimental scoliosis in the rat spine induced by binding the spinous processes. Nihon Seikeigeka Gakkai Zasshi 1994;68:798-807.

6. Lu DS, Luk KD, Lu WW, Cheung KM, Leong JC. Spinal flexibility increase after chymopapain injection is dose dependent: a possible alternative to anterior release in scoliosis. Spine (Phila Pa 1976) 2004;29:1238.

7. Braun JT, Akyuz E, Ogilvie JW. The use of animal models in fusionless scoliosis investigations. Spine (Phila Pa 1976) 2005;30(17 Suppl):S35-45.

8. Rooney GE, Vaishya S, Ameenuddin S, et al. Rigid fixation of the spinal column improves scaffold alignment and prevents scoliosis in the transected rat spinal cord. Spine (Phila Pa 1976) 2008;33:E914-9.

9. Stokes IA, McBride CA, Aronsson DD. Intervertebral disc changes in an animal model representing altered mechanics in scoliosis. Stud Health Technol Inform 2008;140:273-7.

10. Schmid EC, Aubin CE, Moreau A, Sarwark J, Parent S. A novel fusionless vertebral physeal device inducing spinal growth modulation for the correction of spinal deformities. Eur Spine J 2008;17:1329-35.

11. Schwab F, Patel A, Lafage V, Farcy JP. A porcine model for progressive thoracic scoliosis. Spine (Phila Pa 1976) 2009;34:E397-404.

12. Gorman KF, Breden F. Idiopathic-type scoliosis is not exclusive to bipedalism. Med Hypotheses 2009;72:348-52.

13. Grivas TB, Vasiliadis ES, Kaspiris A, Khaldi L, Kletsas D. Expression of matrix metalloproteinase-1 (MMP- 
1) in Wistar rat's intervertebral disc after experimentally induced scoliotic deformity. Scoliosis 2011;6:9.

14. Silva CA, Guirro RR, Delfino GB, Arruda EJ. Proposal of non-invasive experimental model to induce scoliosis in rats. Rev Bras Fisioter 2012;16:254-60.

15. Kaspiris A, Mikelis C, Heroult M, et al. Expression of the growth factor pleiotrophin and its receptor protein tyrosine phosphatase beta/zeta in the serum, cartilage and subchondral bone of patients with osteoarthritis. Joint Bone Spine 2013;80:407-13.

16. Lamprou M, Kaspiris A, Panagiotopoulos E, Giannoudis PV, Papadimitriou E. The role of pleiotrophin in bone repair. Injury 2014;45:1816-23.

17. Kaspiris A, Chronopoulos E, Grivas TB, et al. Effects of mechanical loading on the expression of pleiotrophin and its receptor protein tyrosine phosphatase beta/zeta in a rat spinal deformity model. Cytokine 2016;78:7-15.

18. Aulisa AG, Pola E, Papaleo P, Galli M, Aulisa L. The association between IL-6 and MMP-3 gene polymorphisms and adolescent idiopathic scoliosis: a casecontrol study. Scoliosis 2009;(Suppl 4): O1. https://doi. org/10.1186/1748-7161-4-S1-O1.
19. Jiang J, Qian B, Mao S, et al. A promoter polymorphism of tissue inhibitor of metalloproteinase- 2 gene is associated with severity of thoracic adolescent idiopathic scoliosis. Spine (Phila Pa 1976) 2012;37:417.

20. Jia Y, Liu Y, Tian R, Xu T, Fang Z. The association study of MMP2 polymorphisms with susceptibility to adolescent idiopathic scoliosis in Han Chinese. Int J Clin Exp Pathol 2016;9:10714-20.

21. Dong Z, Nemeth JA, Cher ML, Palmer KC, Bright RC, Fridman R. Differential regulation of matrix metalloproteinase-9, tissue inhibitor of metalloproteinase-1 (TIMP-1) and TIMP-2 expression in cocultures of prostate cancer and stromal cells. Int J Cancer 2001;93:507-15.

22. Lee JS, Suh KT, Eun IS. Polymorphism in interleukin-6 gene is associated with bone mineral density in patients with adolescent idiopathic scoliosis. J Bone Joint Surg Br 2010;92:1118-22.

23. Burner WL 3rd, Badger VM, Sherman FC. Osteoporosis and acquired back deformities. J Pediatr Orthop 1982;2:383-5. 